\title{
A Curvelet-SC Recognition Method for Maize Disease
}

\author{
Jing Luo, ${ }^{1,2}$ Shuze Geng, ${ }^{1,2}$ Chunbo Xiu, ${ }^{1,2}$ Dan Song, ${ }^{1,2}$ and Tingting Dong ${ }^{1,2}$ \\ ${ }^{1}$ Key Laboratory of Advanced Electrical Engineering and Energy Technology, Tianjin 300387, China \\ ${ }^{2}$ College of Electrical Engineering and Automation, Tianjin Polytechnic University, Tianjin 300387, China \\ Correspondence should be addressed to Shuze Geng; gengshuze1234@163.com
}

Received 20 October 2014; Revised 15 March 2015; Accepted 17 March 2015

Academic Editor: William Sandham

Copyright (C) 2015 Jing Luo et al. This is an open access article distributed under the Creative Commons Attribution License, which permits unrestricted use, distribution, and reproduction in any medium, provided the original work is properly cited.

\begin{abstract}
Because the corn vein and noise influence the contour extraction of the maize leaf disease, we put forward a new recognition algorithm based on Curvelet and Shape Context (SC). This method can improve the speed and accuracy of maize leaf disease recognition. Firstly, we use Seeded Regional Growing (SRG) algorithm to segment the maize leaf disease image. Secondly, Curvelet Modulus Correlation (CMC) method is put forward to extract the effective contour of maize leaf disease. Thirdly, we combine CMC with the SC algorithm to obtain the histogram features and then use these features we obtain to calculate the similarities between the template image and the target image. Finally, we adopt $n$-fold cross-validation algorithm to recognize diseases on maize leaf disease database. Experimental results show that the proposed algorithm can recognize 6 kinds of maize leaf diseases accurately and achieve the accuracy of $94.446 \%$. Meanwhile this algorithm has guiding significance for other diseases recognition to an extent.
\end{abstract}

\section{Introduction}

In recent years, maize leaf diseases have a great influence on the yield of maize and quality. Therefore, an important problem caused by them on agriculture shows that it is urgent to protect maize from these diseases and recognize the species of those diseases. There are 6 common maize diseases: Rust spots, Leaf blight, Gray leaf spot, Brown patch, Curvularia leaf spot, and Small spot disease. Their images are shown in Figure 1. At present, the extracted disease features include color features [1], texture features, and shape features [2-5]. The color features include RGB color feature, HIS color feature, RGB color histogram, the standard deviation, the mean, the variance, and the correlation of RGB. The color features are easily influenced by external environmental light, which makes the disease recognition difficult. The color features of diseases are suitable for transient diseases segmentation but not suitable for long-term diseases segmentation. The texture features include the inertia value of gray level cooccurrence matrix, norm of the wavelet transform coefficient, chromaticity moments, and color cooccurrence texture. Compared with the color features of diseases, texture features are not easily affected by external light and are more stable for diseases recognition. The shape features include the area, perimeter, roundness, eccentricity, and rectangle degree. The shape features are suited to some conditions where the diseases have obvious shape features and the edge between disease and healthy tissue is clear. The shape features matching and recognition algorithms based on the transform domain are invariant in photometric transformation, convolution transformation, and rotation transformation, which are not affected by the external environment and perform stably [6] Correspondingly, the disease recognition methods involve Artificial Neural Network (ANN) method [7, 8], Support Vector Machine (SVM) method [9], and Template Match (TM) method [5]. As for ANN method and SVM method, with the increase of the number of samples, the disease recognition model has become more complex, the network structure and the model parameters become more complicated, and the recognition precision and its speed will be affected.

Huang et al. [5] proposed a Principal Component Analysis-Shape Context (PCA-SC) method that combines SC and Principal Component Analysis (PCA). The algorithm transforms the characteristic matrix extracted by SC algorithm into the corresponding covariance matrix firstly. Secondly, PCA algorithm is used to reduce the dimension of the covariance matrix according to the characteristic value order from big to small so as to form a new feature matrix to 


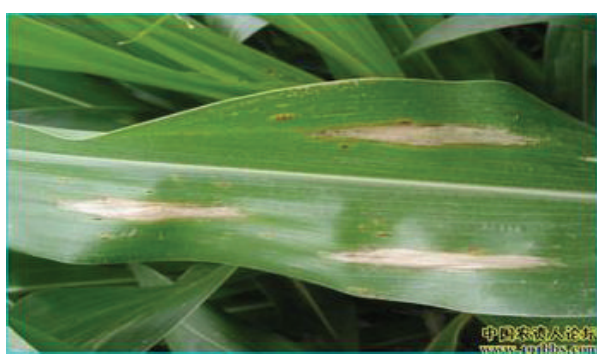

(a) Leaf blight

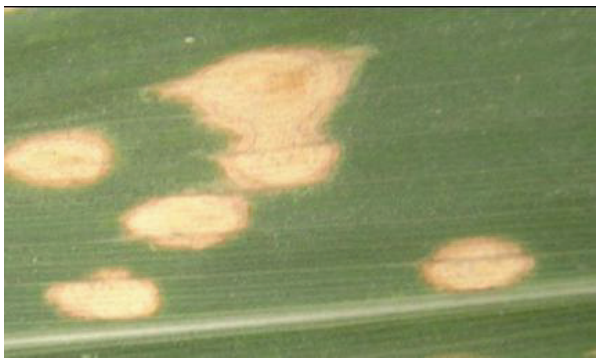

(c) Gray leaf spot

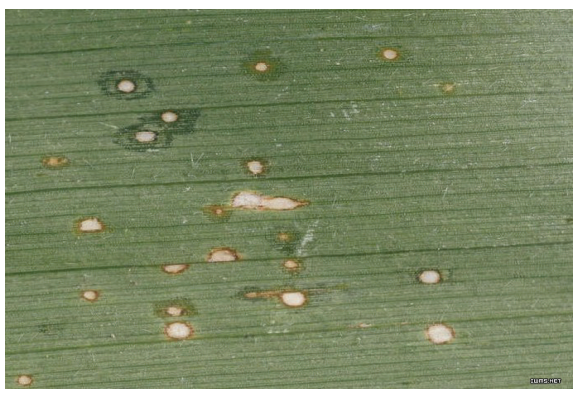

(e) Curvularia leaf spot

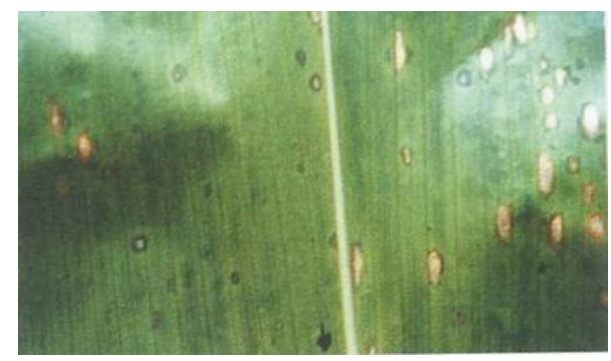

(b) Brown spot

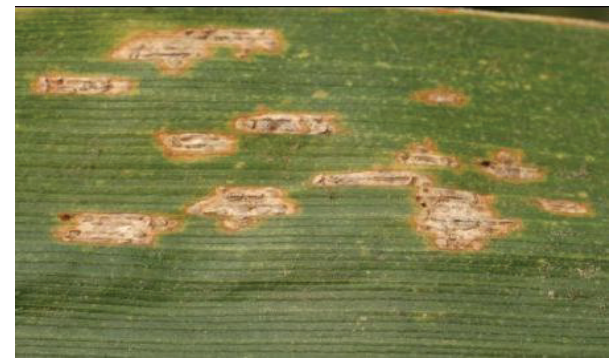

(d) Rust spot

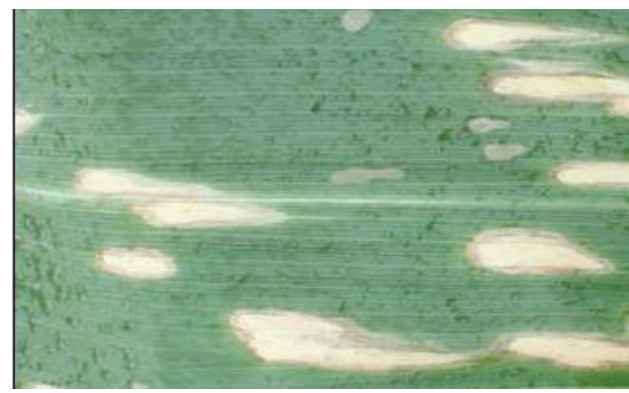

(f) Small spot

FIgURE 1: Maize leaf diseases images.

implement shape matching and object recognition. The algorithm has strong anti-interference ability, high recognition rate, and high recognition accuracy. But it is hard to identify the similar edge.

Target texture edge feature can be described more accurately by Curvelet in multiscale and multidirection [9-11]. Based on discussion above, this paper puts forward a maize leaf diseases recognition mode which is based on Curvelet and SC for these 6 common diseases of maize: Rust spots, Leaf blight, Gray leaf spot, Brown patch, Curvularia leaf spot, and Small spot disease. First of all, this paper puts forward CMC method to realize the extraction of the effective contour of maize leaf disease. Then we combine CMC with the SC algorithm [5] to obtain the histogram features of this disease image and use the matching degree between the target image shape histogram and the template image shape histogram to recognize the disease. Finally, we adopt $n$-fold cross-validation algorithm to recognize diseases on maize leaf disease database. Experimental results show that the algorithm we put forward can recognize those maize leaf diseases accurately. Therefore, this algorithm has guiding significance for maize leaf disease recognition.

\section{Maize Leaf Disease Recognition Method}

2.1. Disease Image Graying. In order to improve the speed of maize leaf disease recognition, we need to transform the color disease image to gray disease image. According to the sensitivity of human eyes to the image, we adopt formula (1) for image transformation. Consider

$$
f(i, j)=0.3 R(i, j)+0.59 G(i, j)+0.11 B(i, j) .
$$

2.2. Disease Image Segmentation. This paper adopts the SRG [12-14] to segment the maize leaf disease image. The basic principles of SRG algorithm are as follows.

Step 1. According to the relationship between the image pixels, the set $S$ of seeds is selected.

Step 2. The set $S$ is marked as $M\left(M_{1}, M_{2}, M_{3}, \ldots, M_{i}\right.$, $\left.\ldots, M_{n}\right)$. Seeds of the group are placed on the stack.

Step 3. The threshold $T_{i}$ of growth center is calculated with Otsu, and $R$ is used for the termination condition of region growing. 


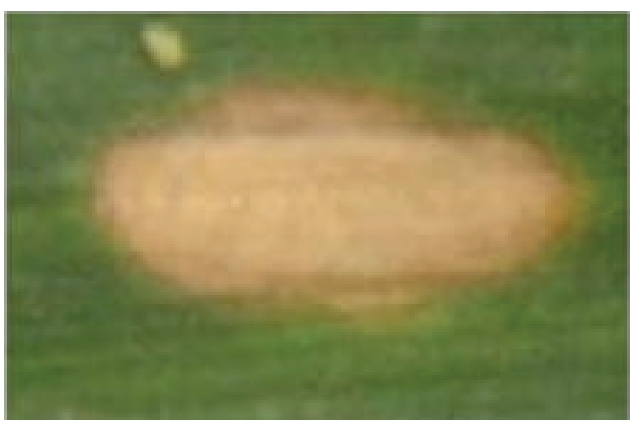

Figure 2: Maize leaf disease original image.

Step 4. Bottom pointer and top pointer of stack are compared, and the seeds at the bottom of stack are selected as the seeds of region growing when bottom pointer is less than top pointer.

Step 5. The points are added into the seeds $M$ when threshold is less than termination condition $\left(T_{i}<R\right)$ in the four neighborhoods of the seed points.

Step 6. Steps 4 and 5 are repeated until $T_{i}>R$.

Maize leaf disease image is shown in Figure 2, and the gray image of maize leaf disease is shown in Figure 3. The segmentation result of Figure 3 is shown in Figure 4.

2.3. Contour Extraction of Maize Leaf Disease Using Curvelet Modulus Correlation. Compared with wavelet, target texture feature can be described more accurately by Curvelet in multiscale and multidirection. In order to reduce the interference of the contour extraction of disease image, the CMC method which is used for obtaining the disease contour is presented. The method is based on the amplitude of the target edge that is large at adjoining scales, while the amplitude of the noise edge can attenuate rapidly at adjoining scales. According to the size of the maize leaf disease, we divide the image into 5 scales. The correlated coefficient between the third scale and the second scale is described as

$$
\operatorname{cor}(S, N)(i, j)=B(S, N)(i, j) \times B\left(S-1, N^{\prime}\right)\left(i^{\prime}, j^{\prime}\right) \text {, }
$$

where

$$
\begin{gathered}
S=3, \quad N=1, K, P_{3}, \\
N^{\prime}=\left[\frac{N}{2}\right], \quad N^{\prime}=1, \ldots, P_{2}, \\
i^{\prime}=\left[\frac{i}{2}\right], \quad i^{\prime}=1, \ldots, 16 \text { or } 18, \\
i=1, \ldots, 32 \text { or } 34 ; \quad j^{\prime}=j=22 .
\end{gathered}
$$

$B(N, S)$ is the Curvelet coefficient at direction $N$ and scale $S$. $P_{3}$ is the direction number of the third scale. $P_{2}$ is the direction number of the second scale.

The concrete implementation steps are as follows.

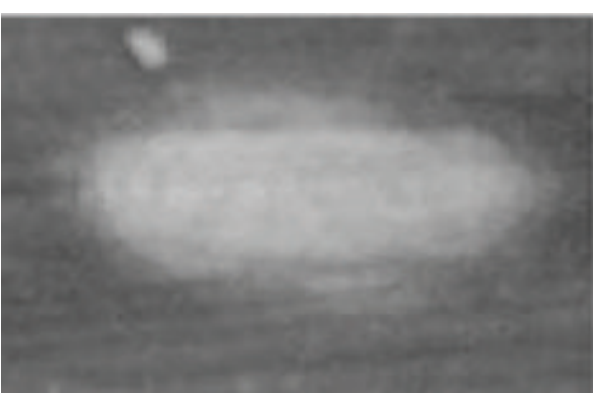

Figure 3: Gray maize leaf disease image.

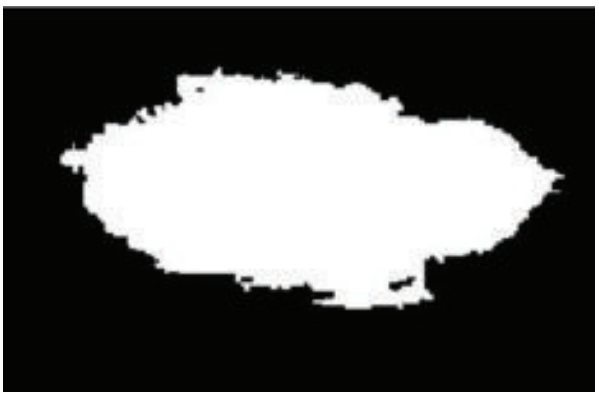

FIgURE 4: Segmentation result of maize leaf disease.

Step 1. The correlated modulus between the third scale and the second scale is calculated.

Step 2. Formula (4) is adopted to detect the edge points of the third scale:

$$
|\operatorname{cor}(S, 2 N-1)(i, j)-\operatorname{cor}(S, 2 N)(i, j)|>T_{s}, \quad s=3,
$$

where $T_{s}$ is the threshold.

Step 3. The following rules are used to detect the edge points at the second scale.

If there are 3 or more than 3 correlation coefficient matrices belonging to the edge, the corresponding matrix is the edge. Otherwise, it is noise.

Step 4. The following rules are used to detect the edge points of the first scale, the fourth scale, and the fifth scale:

$$
|B(S, N)|>P_{s}, \quad s=1,4,5,
$$

where $P_{s}$ is the threshold value.

Step 5. The edge points of each scale are reconstituted to accomplish the edge detection.

Figure 6 is the detection result of Figure 5. This result shows that CMC method can extract the disease contour clearly. It is good for subsequent disease feature extraction.

2.4. Feature Extraction of Maize Leaf Disease. The SC method presented by Belongie [15] is an operator which is used for describing the shape. Its core idea is point set matching. 


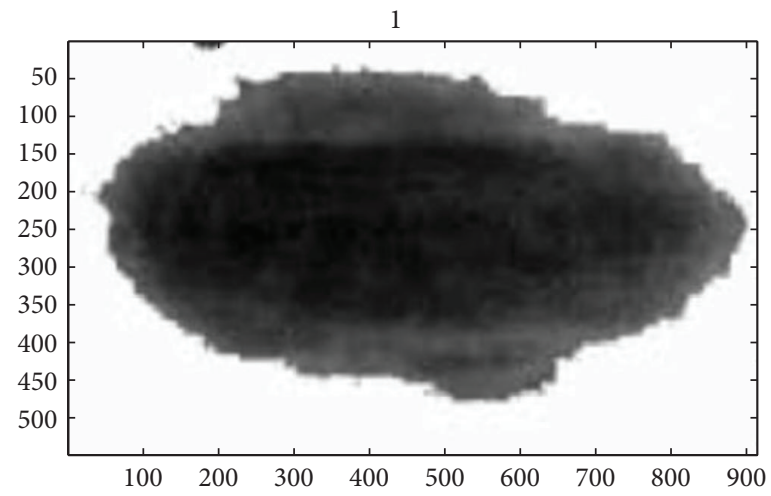

Figure 5: Segmentation result of Gray leaf spot.

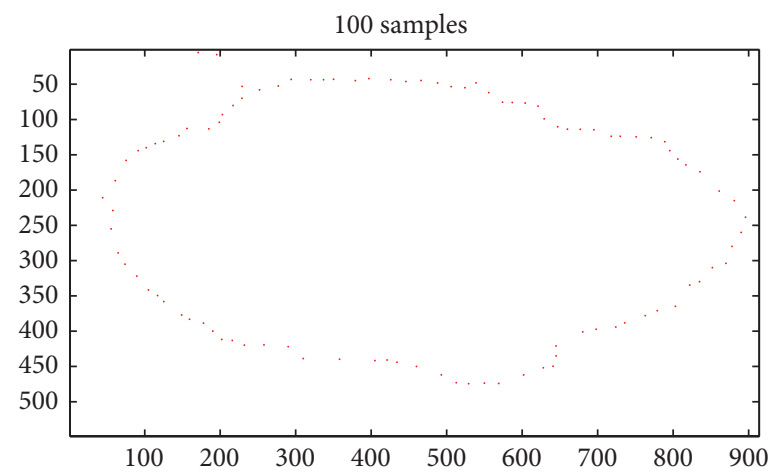

FIGURE 6: Detection result of Figure 5 by CMC.

The flow of maize leaf disease recognition based on SC is as follows.

Step 1. The obtained edge detecting point set $(x, y)$ is transformed into $\log$-polar $(\log r, \theta)$.

The transformation relation between rectangular coordinates and log-polar is as follows:

$$
r=\sqrt{x^{2}+y^{2}} ; \quad \rho=\log \left(\sqrt{x^{2}+y^{2}}\right) ; \quad \theta=\arctan \frac{y}{x} .
$$

\section{Step 2. The points are calculated in log-polar.}

The obtained result in the polar coordinate is transformed into 12 directions, and each direction is 30 degrees. $\log _{2} r$ is the step, and each direction is divided into 5 portions. So the whole space is divided into 60 bins. For a fixed point of the contour called $p_{i}$, its property is described by the number of pixels in each bin. Thus, every feature point can be described by a row vector of 60 dimensions (characteristic attributes). If the number of the edge points is $n$, the disease feature can be described by a matrix $(n \times 60)$. In this paper $n$ equals 100 .

Figure 7 shows the log-polar coordinate.

Step 3. The similarity between the template image and the target image is calculated.
The similarity between the point $P_{i}$ of the model image and the point $Q_{j}$ of the target image is calculated by

$$
C_{i j}=C\left(P_{i}, Q_{j}\right)=\frac{1}{2} \sum_{k=1}^{K} \frac{\left[h_{i}(k)-h_{j}(k)\right]^{2}}{h_{i}(k)+h_{j}(k)},
$$

where $h_{i}(k)$ is the corresponding histogram of point $P_{i}, h_{j}(k)$ is the corresponding histogram of point $Q_{j}$, and $K=60$.

If $C_{i j}$ is smaller, the point $P_{i}$ and the point $Q_{j}$ are more matching.

Thus, formula (8) is used to calculate the similarity between the model image and the target image. Consider

$$
C=\frac{\sum_{i=1}^{n}\left(\sum_{j=1}^{m} C_{i j}\right) / m}{n} .
$$

If the number of target disease image contours is $n$ and the number of model disease image contours is $m$, we can get $n \times m$ matching. In this paper, the threshold $T_{1}$ is set to 50 . If the matched-degree $C$ is less than $T_{1}$, the model image and the target image are matched; otherwise, the model image and the target image are not matched.

Figure 8 shows a feature point of 60 characteristic attributes in the log-polar coordinates.

Figure 9 shows 100 -feature extraction of the Gray leaf spot disease (Figure 6). Each feature point has 60 attributes, where 


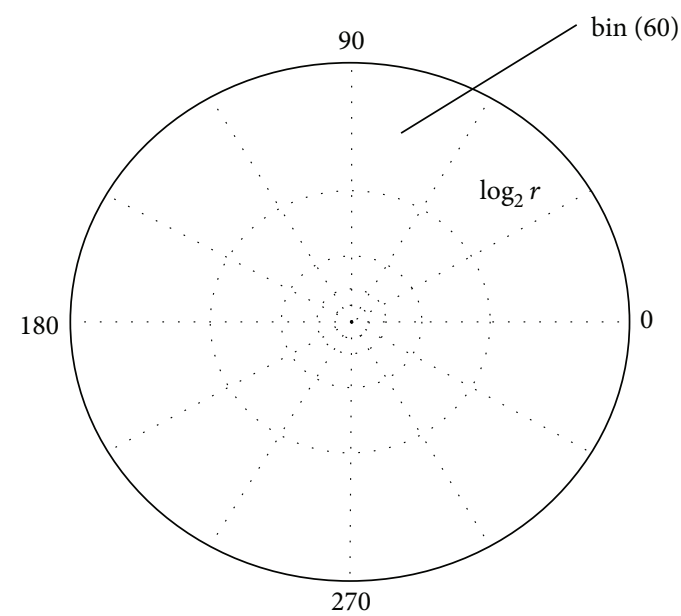

FIgURE 7: Schematic diagram of log-polar coordinate.

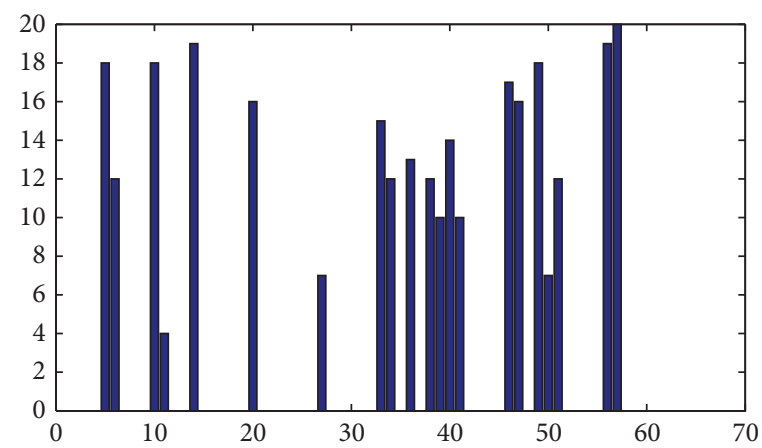

FIGURE 8: 60 characteristic attributes of a feature point.

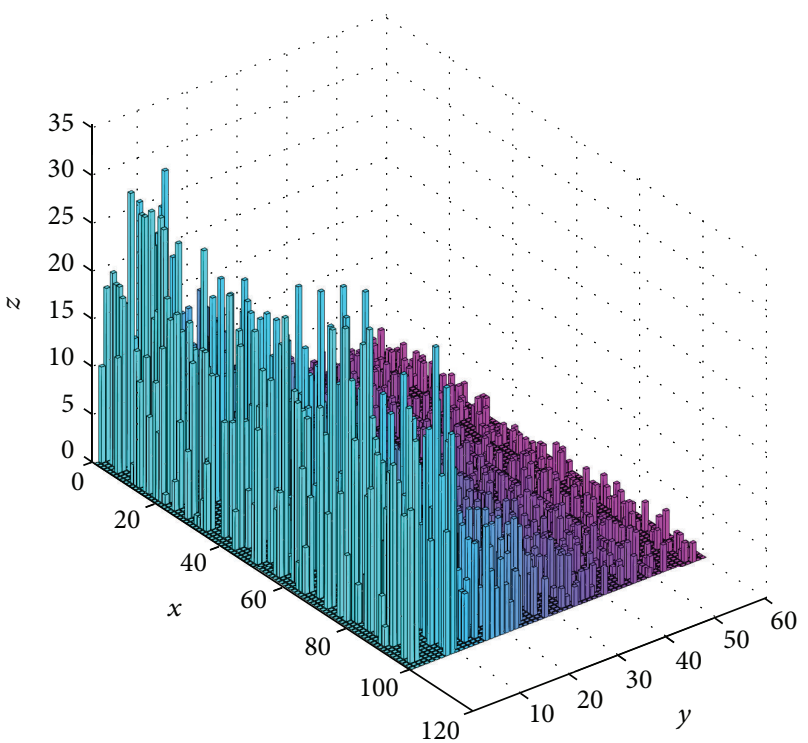

FIGURE 9: Feature points and characteristic attributes. 


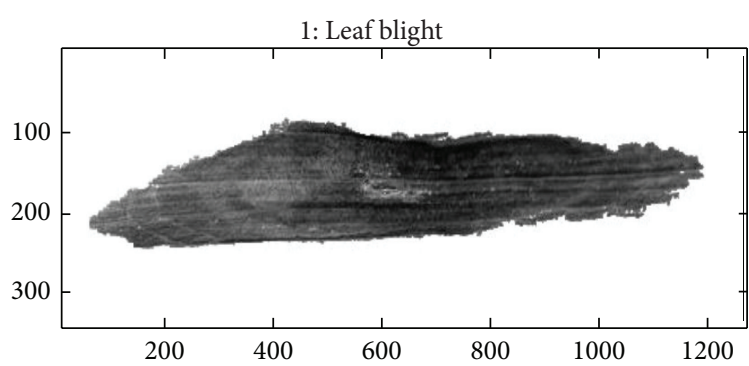

(a)

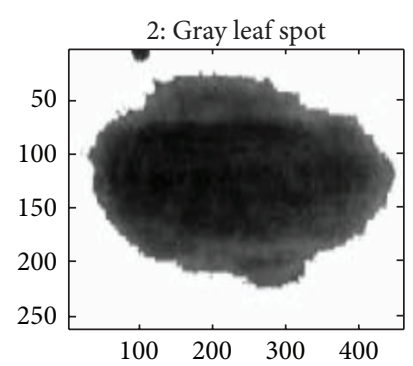

(b)

FIGURE 10: Leaf blight and Gray leaf spot segmentation results.
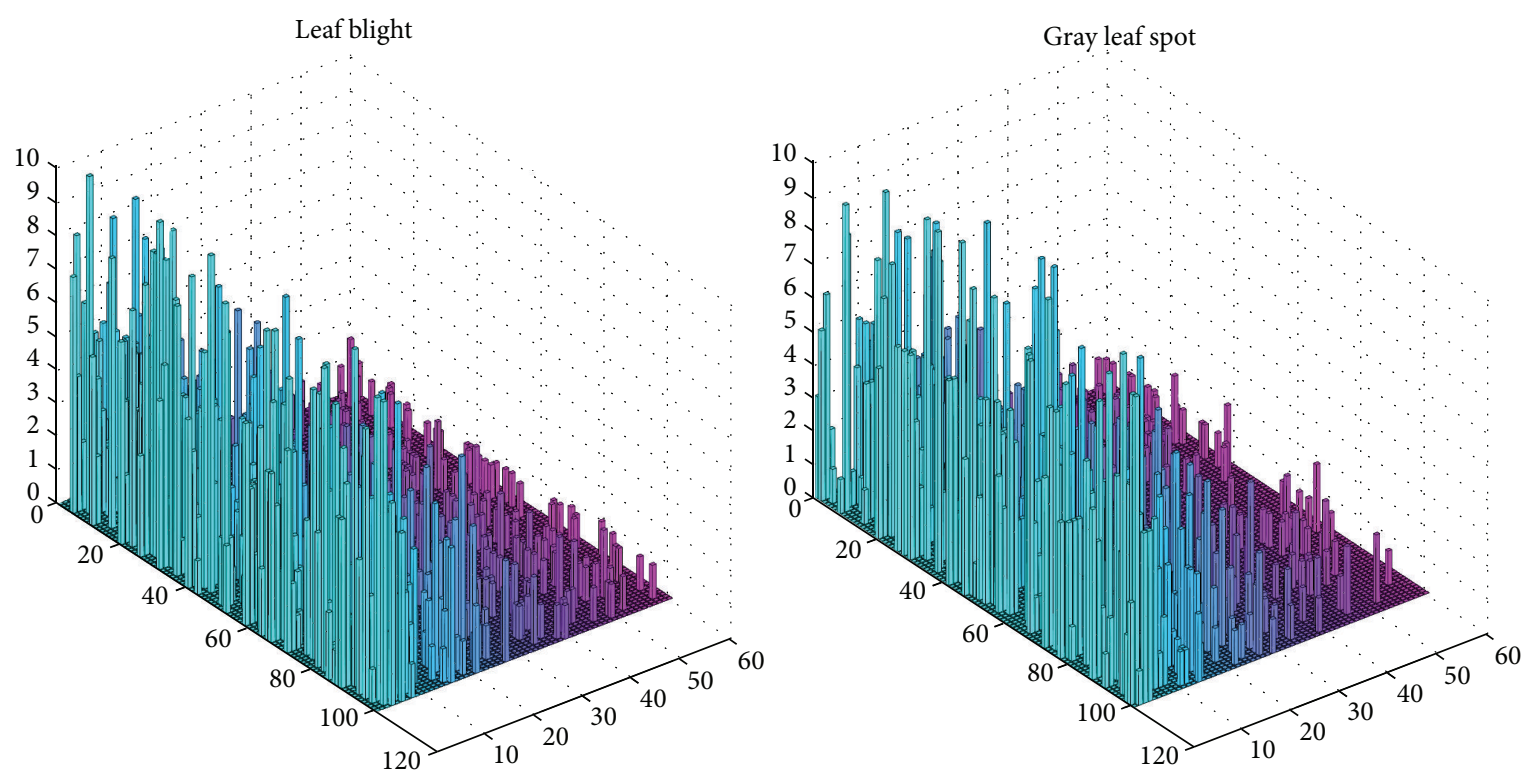

FIGURE 11: Feature extraction results of Gray leaf spot and Leaf blight disease.

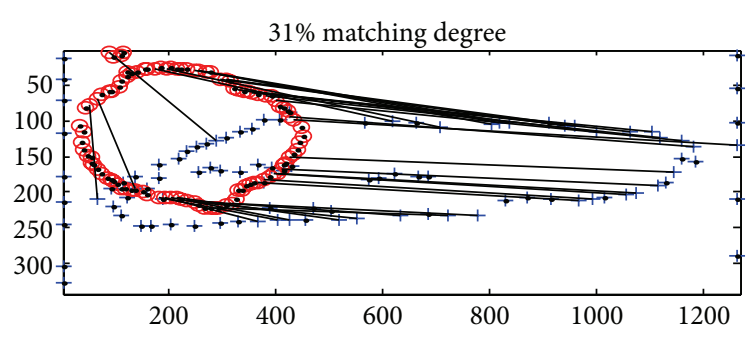

FIGURE 12: The final matching results.

$x$ represents the number of feature points and $y$ represents 60 bins. $z$ represents the number of pixels of each bin.

The Leaf blight and Gray leaf spot segmentation and feature extraction are shown in Figures 10 and 11. 10(b).

Figure 12 shows the matching results of Figures 10(a) and

From Figure 12, the matching results are 31\% $(31 \%<$ $50 \%)$; the algorithm can distinguish between the Leaf blight and the Gray leaf spot disease.

\section{Experimental Results and Analysis}

Experimental environment is as follows: the operating system is windows 8, Pentium (R), quad core, $8 \mathrm{G}$ internal storage, and the software is matlabR2011b. The experimental images are from the maize leaf disease database established by Institute of Crop Science, Chinese Academy of Agricultural Sciences.

3.1. Experimental Subjects. 744 images are selected to verify the accuracy of the proposed algorithm. Every disease responds to 124 images, and there are 6 kinds of diseases. There are four templates for each type of disease and 120 test samples. Figure 13 shows four templates of Brown spot.

3.2. Experimental Results. In the experiment, 6-fold cross validation is used to train and test the proposed algorithm. The 744 plant disease images are divided into 6 independent subsets, and each subset contains 124 plant disease images. Each subset has 4 template images and 120 training samples. The training and testing process is repeated 6 times, and finally the 6-time test results are averaged. The recognition 

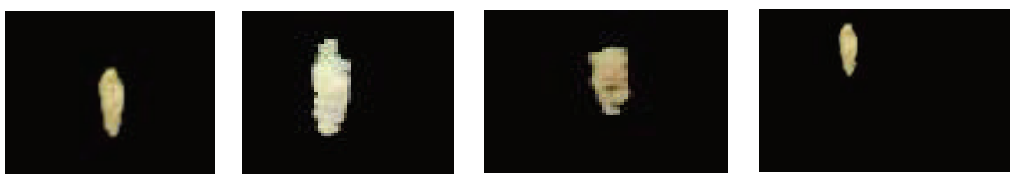

FIGURE 13: Brown spot samples.

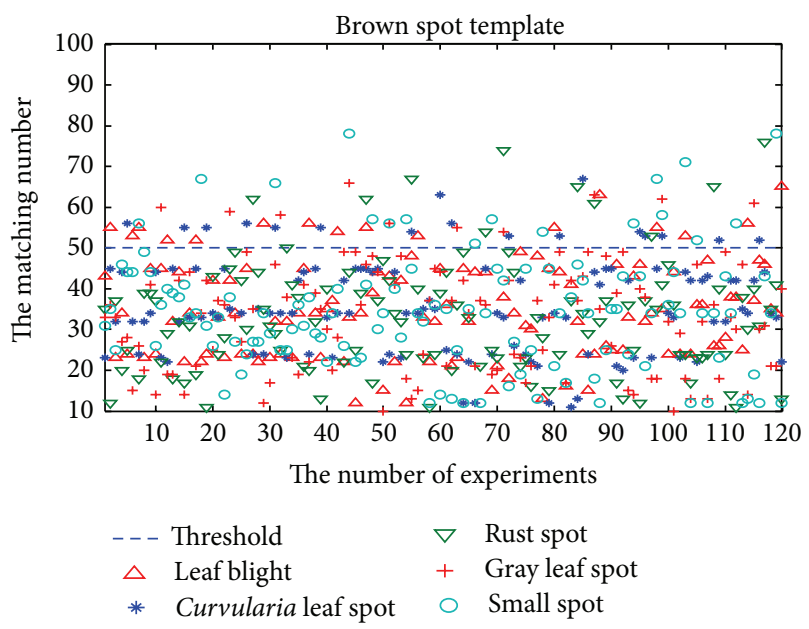

FIGURE 14: Experiment results between different kinds of diseases.

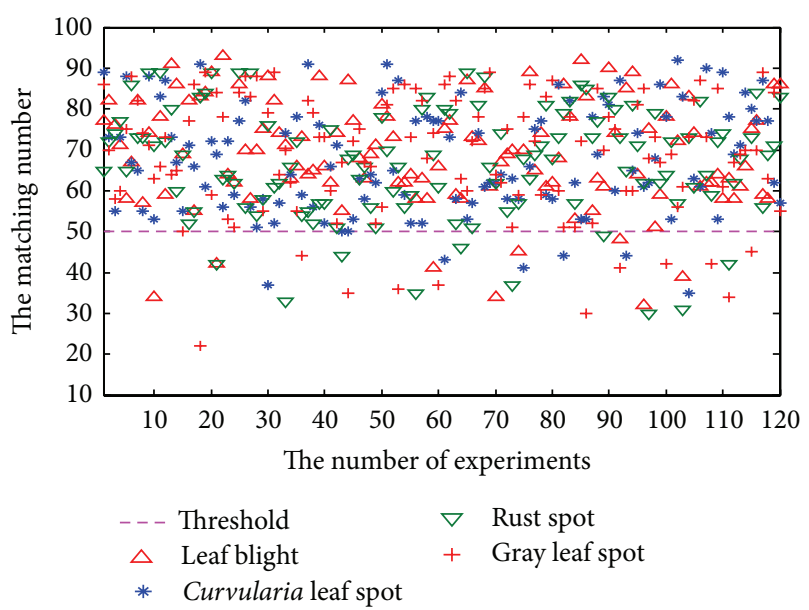

FIGURE 15: Experiment results between the same diseases.

rate between the same kinds of diseases and different kinds of diseases is calculated, respectively.

Figure 14 shows the results between different kinds of diseases. The Brown spot is selected as template. The remaining 5 kinds of diseases are used as test samples.

Figure 15 shows results between the same diseases. The Leaf blight, Gray leaf spot, Rust spot and Curvularia leaf spot are selected as templates. The corresponding test samples are used as test samples. For the same diseases between experiments.

Figure 16 shows recognition rate between the same diseases corresponding to 4 templates.

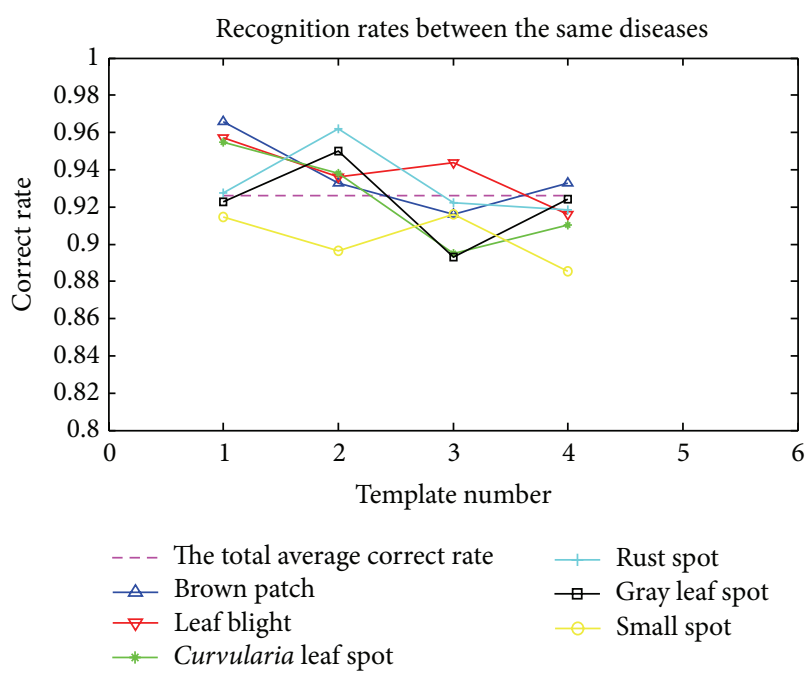

Figure 16: Recognition rates between the same diseases.

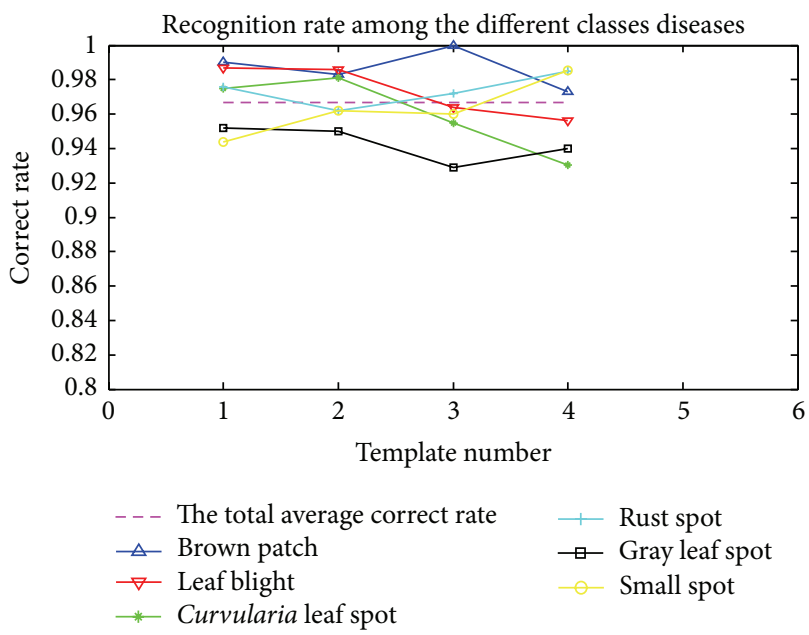

Figure 17: Recognition rate among the different classes diseases.

Figure 17 shows recognition rate among the different classes diseases corresponding to 4 templates.

From the above, the results are shown in Table 1.

From the experiment results, recognition rates between the same diseases and recognition rate among the different classes diseases reach above 90\%. In the same diseases recognition, the recognition rate of Brown patch is the highest (97.385\%), and the Small spot disease has the lowest recognition rate $(88.673 \%)$. In the different classes diseases recognition, Brown patch has the highest recognition rate $(98.658 \%)$, and Gray leaf spot has the lowest recognition rate $(93.434 \%)$. 
TABLE 1: Maize leaf disease recognition accuracy (\%).

\begin{tabular}{lcc}
\hline $\begin{array}{l}\text { Recognition rates } \\
\text { between the same } \\
\text { diseases }\end{array}$ & $\begin{array}{c}\text { Recognition rate } \\
\text { among the different } \\
\text { classes diseases }\end{array}$ & $\begin{array}{c}\text { The total recognition } \\
\text { rate }\end{array}$ \\
\hline $92.225 \%$ & $96.667 \%$ & $94.446 \%$ \\
\hline
\end{tabular}

Some edges extracted from Gray leaf spot and Curvularia leaf spot are similar, some edges extracted from Small spot and Gray leaf spot are also similar, and some edges extracted from Brown patch, Curvularia leaf spot, and Gray leaf spot are also similar. These similarities lead to the error recognition.

\section{Conclusion}

Because the corn vein and noise influence the contour extraction of the maize leaf disease, we put forward a new maize leaf disease recognition algorithm using the Curvelet technique and SC. This method can improve the speed of maize leaf disease recognition. The Curvelet-SC descriptor was put forward based on SC algorithm and the principle of this algorithm was elaborated in the paper. It combined Curvelet and SC for shape matching and texture recognition.

With the experimental analysis, the average accuracy of Curvelet-SC descriptor for maize leaf disease recognition is up to $94.446 \%$. Thus, Curvelet-SC shape descriptor is an efficient method for disease recognition. The proposed algorithm can recognize the maize leaf disease, and it also has guiding significance for other disease recognition to an extent.

\section{Conflict of Interests}

The authors declare that there is no conflict of interests regarding the publication of this paper.

\section{Acknowledgments}

The authors are grateful to the anonymous reviewers who made constructive comments. This work is supported by the National Natural Science Foundation of China (nos. 61203302 and 51107088) and the Tianjin Research Program of Application Foundation and Advanced Technology (no. 14JCYBJC18900).

\section{References}

[1] X. Hu, B. Li, and F. Liu, "Image segmentation based on graph theory in multi-color space for maize leaf disease," Transactions of the Chinese Society for Agricultural Machinery, vol. 44, no. 2, pp. 177-181, 2013.

[2] D. C. Zheng and M. Han, "Based on the context of H-EMD shape characteristic shape matching method," Control and Decision, vol. 27, no. 11, pp. 1639-1643, 2012.

[3] S. Yang, "Spectra of shape contexts: an application to symbol recognition," Pattern Recognition, vol. 47, no. 5, pp. 1891-1903, 2014.

[4] M. Han and D. C. Zheng, "Shape recognition based on fuzzy shape context," Acta Automatica Sinica, vol. 38, no. 1, pp. 68-75, 2012.
[5] W.-G. Huang, C. Gu, and Z.-K. Zhu, "PCA-SC shape matching for object recognition," Optics and Precision Engineering, vol. 21, no. 8, pp. 2103-2110, 2013.

[6] H. Yang and Q. Wang, "A novel local invariant feature detection and description algorithm," Chinese Journal of Computers, vol. 33, no. 5, pp. 935-944, 2010.

[7] J.-L. Buessler, P. Smagghe, and J.-P. Urban, "Image receptive fields for artificial neural networks," Neurocomputing, vol. 144, no. 20, pp. 258-270, 2014.

[8] A. Goltsev and V. Gritsenko, "Investigation of efficient features for image recognition by neural networks," Neural Networks, vol. 28, pp. 15-23, 2012.

[9] J. Luo, D. Song, C. Xiu, S. Geng, and T. Dong, "Fingerprint classification combining Curvelet transform and gray-level cooccurrence matrix," Mathematical Problems in Engineering, vol. 2014, Article ID 592928, 15 pages, 2014.

[10] X. Feng, X. Wang, and J. Dang, "Image texture classification based on Curvelet-scattering features," Transactions of the Chinese Society of Agricultural Machinery, vol. 43, no. 10, pp. 184-199, 2012.

[11] J. Zhao and S. Qu, "The fuzzy nonlinear enhancement algorithm of infrared image based on curvelet transform," Procedia Engineering, vol. 15, pp. 3754-3758, 2011.

[12] S. Zhang, K. Wang, and Z. Liu, "Segmentation algorithm based on discrete cosine transform and region growing image of whitefly," Transactions of the Chinese Society of Agricultural Engineering, vol. 29, no. 13, 2013.

[13] D. Ni and H. Ma, "Multi-source remote sensing image registration based on region growing," Acta Automatica Sinica, vol. 40, no. 6, 2013

[14] W. Gao, J. Shen, and Y. Wang, "Applications based on local adaptive region growing multi-template matching in the automatic detection of retinal hemorrhage," Spectroscopy and Spectral Analysis, vol. 33, no. 2, pp. 448-453, 2013.

[15] S. Belongie, J. Malik, and J. Puzicha, "Shape matching and object recognition using shape contexts," IEEE Transactions on Pattern Analysis and Machine Intelligence, vol. 24, no. 4, pp. 509-522, 2002. 

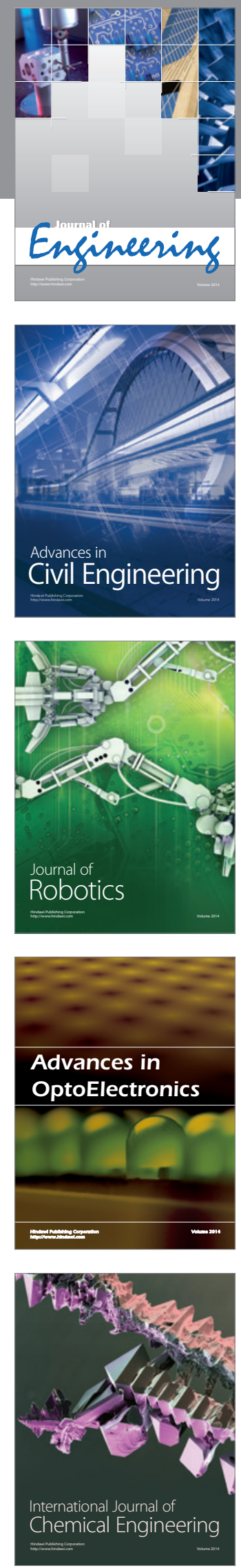

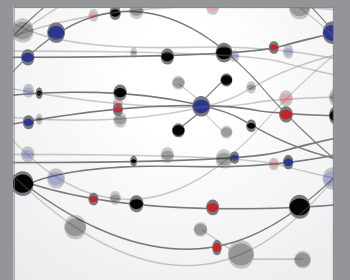

The Scientific World Journal
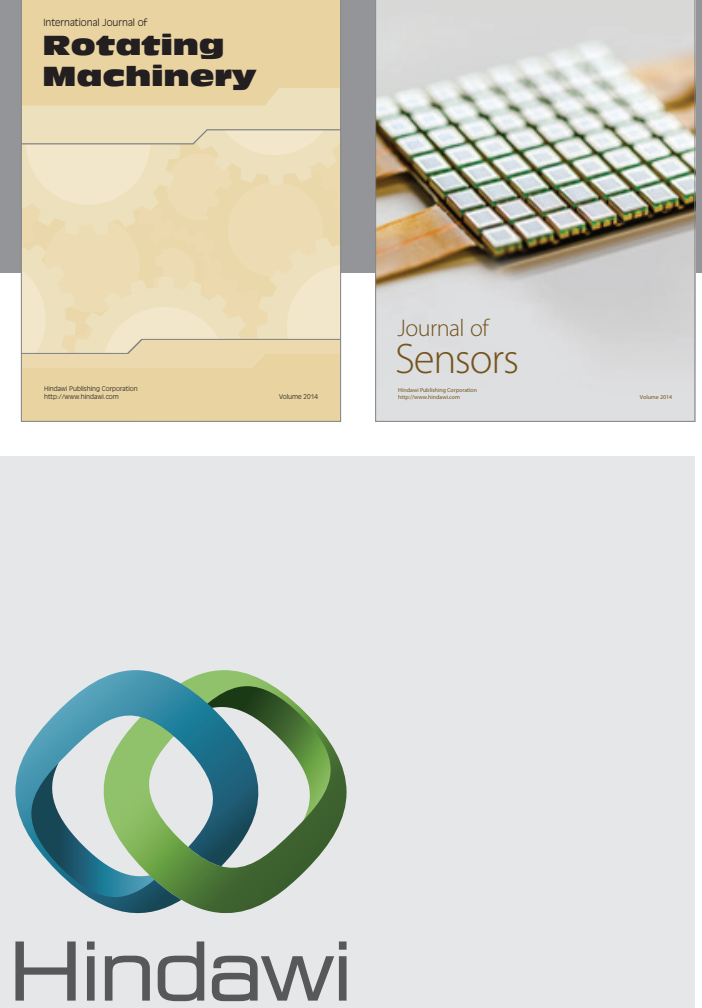

Submit your manuscripts at http://www.hindawi.com
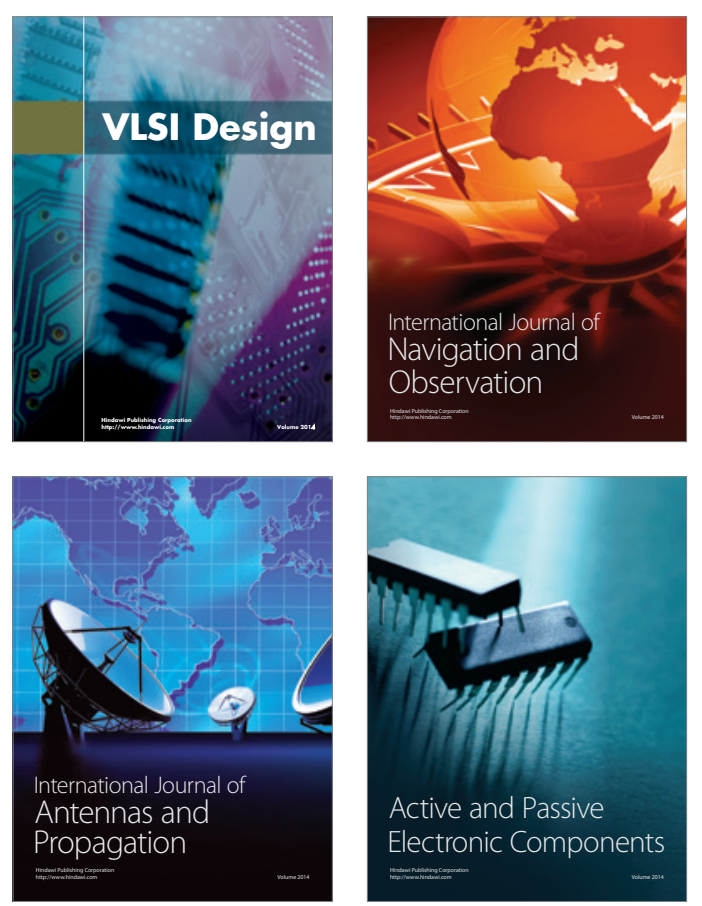
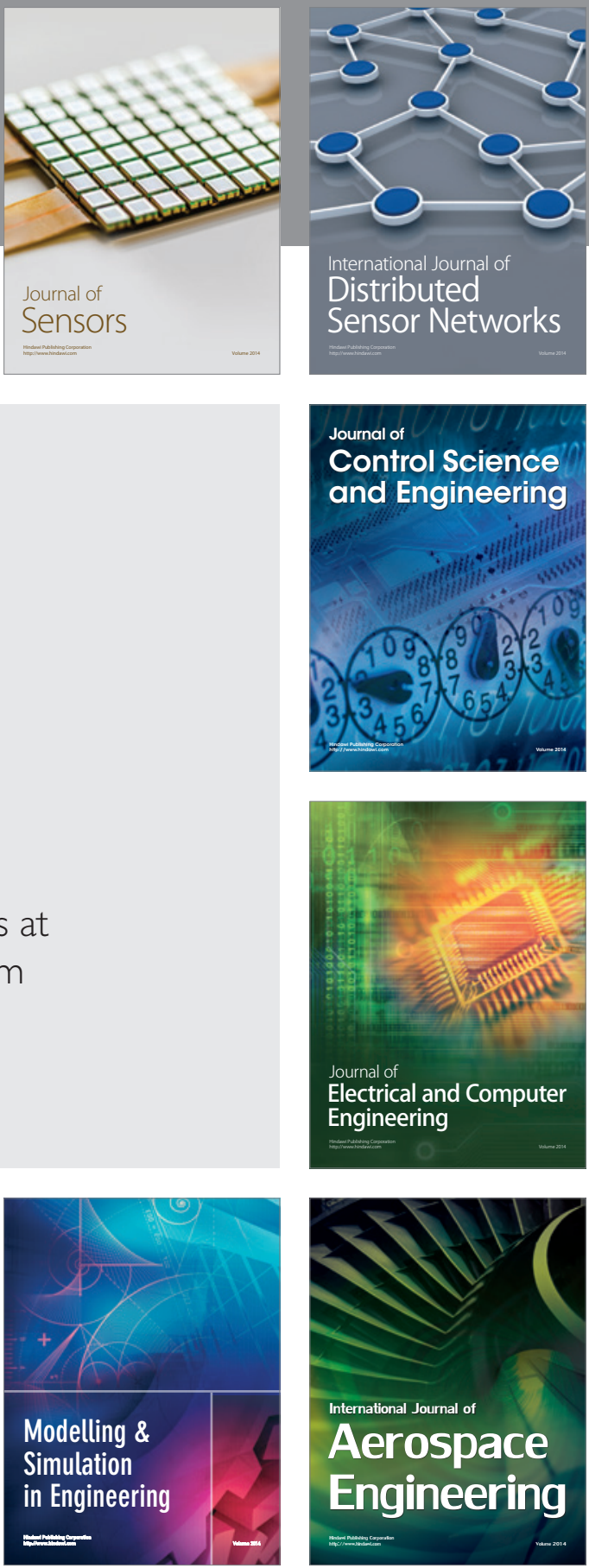

Journal of

Control Science

and Engineering
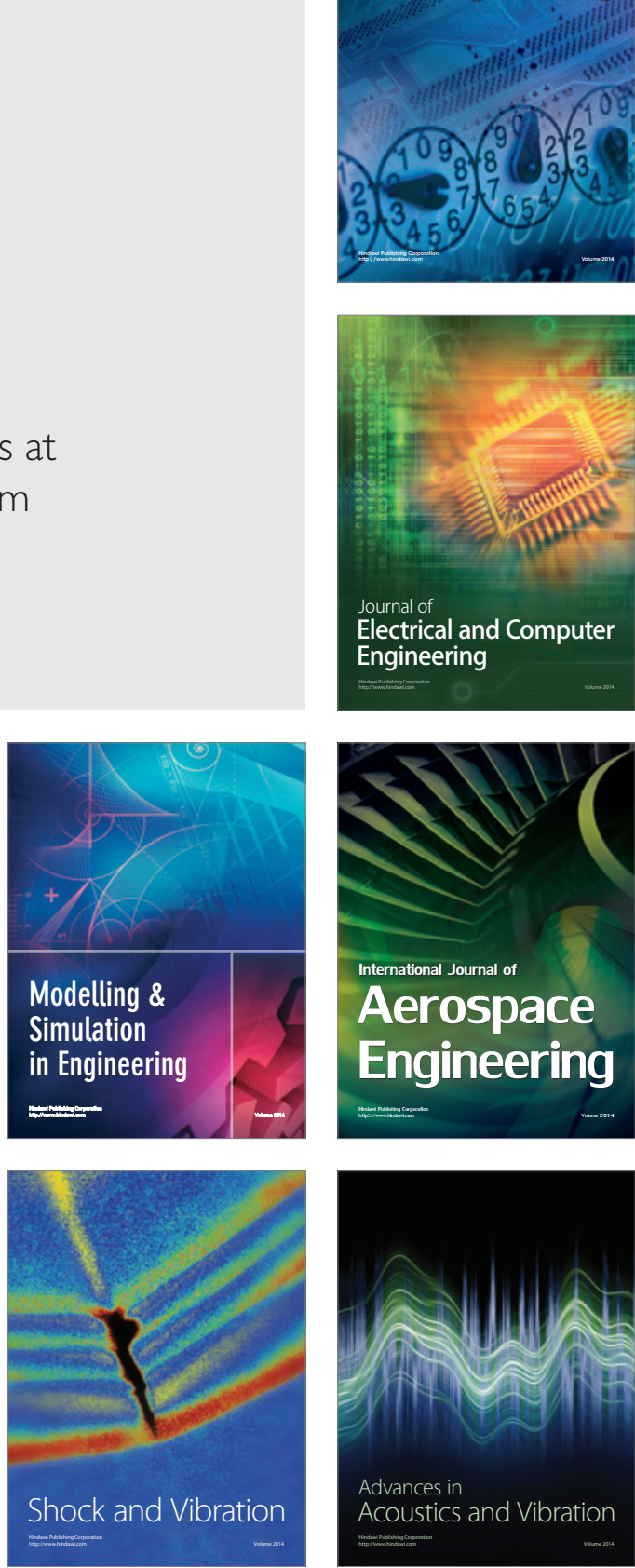